\title{
Surgical pathology in sub-Saharan Africa-volunteering in Malawi
}

\author{
Sabina Berezowska $\cdot$ Tamiwe Tomoka $\cdot$ Steve Kamiza • \\ Danny A. Milner Jr. $\cdot$ Rupert Langer
}

Received: 9 February 2012 / Accepted: 22 February 2012 /Published online: 10 March 2012

(C) Springer-Verlag 2012

\begin{abstract}
The breadth of material found in surgical pathology services in African countries differs from the common spectrum of "the West". We report our experience of a voluntary work in the pathology departments of Blantyre and Lilongwe, Malawi. During a 6-week period, 405 cases (378 histology and 27 cytology cases) were processed. The vast majority showed significant pathological findings $(n=$ 369 ; $91.1 \%$ ): 175 cases $(47.4 \%)$ were non-tumoral conditions with predominance of inflammatory lesions, e.g., schistosomiasis $(n=11)$ and tuberculosis $(n=11)$. There were $39(10.6 \%)$ benign tumors or tumor-like lesions. Intraepithelial neoplasia of the cervix uteri dominated among premalignant conditions $(n=15 ; 4.1 \%)$. The large group of malignancies $(n=140 ; 37.9 \%)$ comprised 11 pediatric tumors (e.g., rhabdomyosarcoma, small blue round cell tumors) and 129 adult tumors. Among women $(n=76)$,
\end{abstract}

Electronic supplementary material The online version of this article (doi:10.1007/s00428-012-1217-z) contains supplementary material, which is available to authorized users.

S. Berezowska $\cdot$ R. Langer $(\bowtie)$

Institute of Pathology, University of Bern,

Murtenstr 31,

3010 Bern, Switzerland

e-mail: rupert.langer@pathology.unibe.ch

T. Tomoka $\cdot$ S. Kamiza

Department of Histopathology, College of Medicine,

University of Malawi,

Blantyre, Malawi

D. A. Milner Jr.

Department of Pathology, Brigham and Women's Hospital,

Boston, MA, USA

R. Langer

Institute of Pathology, Technische Universität München,

Munich, Germany squamous cell carcinomas (SCCs) of the cervix uteri predominated $(n=25 ; 32.9 \%)$, followed by breast carcinomas $(n=12 ; 15.8 \%)$ and esophageal SCC $(n=9 ; 11.8 \%)$. Males $(n=53)$ most often showed SCC of the esophagus $(n=9$; $17.0 \%)$ and of the urinary bladder $(n=7 ; 13.2 \%)$. Lymphomas $(n=7)$ and Kaposi's sarcomas $(n=6)$ were less frequent. Differences compared to the western world include the character of the conditions in general, the spectrum of inflammatory lesions, and the young age of adult tumor patients (median 45 years; range 18-87 years). Providing pathology service in a low-resource country may be handicapped by lack of personnel, inadequate material resources, or insufficient infrastructure. Rotating volunteers offer a bridge for capacity building of both personnel and the local medical service; in addition, the volunteer's horizons are broadened professionally and personally.

Keywords Pathology - Low-resource country · Africa

\section{Introduction}

Providing pathology service in low-resource countries is challenging due to the lack of trained personal and technical equipment and inefficient infrastructure (e.g., transportation, communication, etc.) [1-3]. The latter of these can be built quickly when money, demand, and motivation are available; the former (i.e., personnel) requires time for training which creates gaps. The challenge of providing high-quality pathological services in Africa is limited by the number of available well-trained and skilled pathologists in a particular country or medical unit. When indigenous highly skilled pathologists are available, they may often be so few in number that the overwhelming challenges of providing medical education, pathology service, and government/ 
administrative responsibilities hinder their day to day signout ability. When a low-resource pathology unit has the opportunity to add staff to their system, they are still faced with the gap between the arrivals of the new team members. One system to overcome this problem which can serve as a temporary bridge while building the required capacity with indigenous pathologists is a rotating volunteer system. This provides qualified pathologist who can insure continuity, establish turn-around-time goals, and train local medical staff in the best methods for tissue collection for both histology and cytology.

Malawi is a small country in southeast Africa bordered by Tanzania in the north and northeast, Zambia in the west, and Mozambique in the southeast and southwest. It is one of the smallest but most densely populated counties in subequatorial Africa with a population of currently around 16 million inhabitants, of whom the vast majority lives in rural areas. Malawi belongs to the poorest countries in the world with around $40 \%$ of Malawians living below the international poverty line. The economy is essentially agricultural, and tobacco accounts for $70 \%$ of Malawi's export earnings, followed by tea and sugar - all mainly grown by smallholder farmers. The country is heavily dependent on foreign aid, which represents half of its gross domestic product [4].

Like most sub-Saharan countries, Malawi suffers from a high burden of HIV, with an estimated prevalence of $15 \%$ for the age group between 15 and 49 years. Tuberculosis and malaria are common [5, 6]. Major efforts to establish and improve Malawi's health care system are in process, e.g., offering the main health services free of charge. Life expectancy has successfully improved from less than 40 years in 2000 to 52.2 years in 2009 [4].

In Malawi, there are currently two functioning government-run pathology laboratories situated in the University of Malawi College of Medicine in Blantyre (serving the majority of the country's hospitals) and at the Kamuzu Central Hospital in Lilongwe (Malawi's capital). There are four fully certified pathologists. One of them does forensic postmortems only and one retired pathologist is providing service for the Lilongwe lab. The remaining two (TT and KS) are working at the College of Medicine. Being faculty members, they are also heavily involved in administrative and academic duties. Two local residents are currently abroad and two more are beginning their residency in 2012. All residents are trained in South Africa over a 4year period, as special education for pathology residents is currently not available in Malawi.

In July 2011, a structured support project for the pathology service of Malawi was initiated by Danny Milner from the Department of Pathology, Brigham and Women's Hospital, Boston, USA, in association with the Partners in Health [7]. The aim of the Pathologists for Malawi Project (which uses the well-established model provided by the long-time operating organization "Pathologists Overseas") is to provide continuous on-site support by volunteer pathologists until local pathology residents finish their training abroad, thereby shortening turnover times, avoiding larger backloads, but also creating an intellectual and cultural exchange between local and foreign pathologists. Here, we report our individual experience of a 6-week voluntary work in the pathology departments of Blantyre and Lilongwe, Malawi, between November and December 2011.

\section{Materials and methods}

Pathology laboratories in Blantyre and Lilongwe

The Department of Histopathology in Blantyre is part of the College of Medicine of Malawi and provides diagnostic service for the large Queen Elisabeth Central Hospital in Blantyre and for at least 20 additional hospitals of Blantyre and the rest of the country. The annual workload reaches around 3,000 cases for histological examination (estimated to be approximately $10 \%$ of the actual potential caseload for the country). Moreover, the department provides pathology service for a large number of scientific projects, mostly coordinated from abroad.

In Lilongwe, the histopathologic laboratory is located within the area of the Kamuzu Central Hospital $(\mathrm{KCH})$, the capital's largest clinic. The lab is set up and financed by the University of North Carolina (UNC), which is running a large research center on the campus. Before the UNC-sponsored lab was constructed, there were approximately 150 cases per year from $\mathrm{KCH}$. In the first 6 months of operation, 700 diagnostic cases processed, creating a projected caseload of $\sim 1,500$ cases annually (a tenfold increase). Because there is no on-site permanent pathologist in Lilongwe, the slides are produced at $\mathrm{KCH}$ but then have to be sent to Blantyre for reading and signing-out by Professor George Liomba, the retired former head of the Blantyre Department of Histopathology. Both laboratories (Blantyre and Lilongwe) are fully functional, equipped with embedding processors, microtomes, and automatic staining machines. At present, hematoxylin and eosin (H\&E), periodic acid-Schiff, and Ziehl-Neelsen (ZN) stains are available. Both labs plan to implement further special histochemical and basic immunohistochemical stains. There are expert technicians on both sites, and additional staff is going to be trained during the next years.

Pathology volunteers

The work of volunteering pathologist mainly consists of providing diagnostic service, which results in immediate support for the local pathologists. Furthermore, there is a 
possibility to assist in teaching of the forthcoming pathology residents (e.g., grossing and histology, preparing teaching slides), technicians, and clinicians (e.g., performing fine needle aspirations). The volunteers are self-funded but housing is provided by Danny Milner and the UNC.

Time period reported

The reported cases encompass our diagnostic workload (SB and RL) during a 6-week period between November and December 2011 while working as volunteering pathologists in Malawi-4 weeks in Blantyre and 2 weeks in Lilongwe.

\section{Results}

A total of 405 cases were processed and reviewed, encompassing 378 histology specimens (139 biopsies, 133 excisions and curettages, and 106 resection specimens) and 27 cytology cases. The Blantyre load included 326 cases, while 79 cases were seen in Lilongwe. The majority of cases $(n=$ $120,29.6 \%$ ) were from gynecological sites (uterus, ovary, and breast), followed by gastrointestinal cases ( $n=73 ; 18 \%$; esophagus and colon). An overview about the distribution of the various sites is given in Fig. 1. Most cases showed significant pathological findings. In some of the 26 cases lacking pathological changes, the clinical diagnosis of a tumor (e.g., stenosing esophageal cancer) implied insufficient sampling. The cases with pathological findings consisted of 175 (47.4\%) non-tumoral conditions, 39 (10.6\%) benign tumors or tumor-like lesions, 15 (4.1\%) premalignant changes, and 140 (37.9\%) malignant tumors. Ten cases (six of them cytology) were technically unsatisfactory.

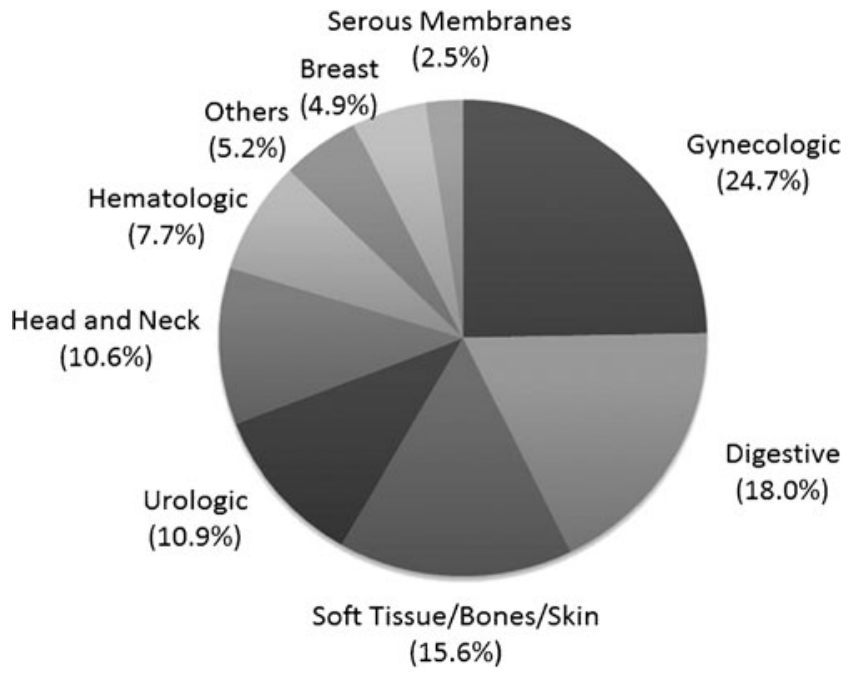

Fig. 1 Topographical distribution of cases
Macroscopic and histologic pictures of some examples of the cases are given in Fig. 2. A complete list of all cases is provided as Electronic supplemental material.

\section{Benign conditions}

Eighty-nine cases $(22.0 \%)$ showed only degenerative or functional changes, like disorders of pregnancy (ectopic pregnancies or missed abortions), Hirschsprung's disease $(n=4)$, cysts, hyperplastic conditions, or fibrosis. Inflammatory conditions as main diagnosis were found in 94 cases $(25.5 \%)$; the most frequent sites being soft tissue, cervix, uteri, and appendix. Unspecific inflammation was acute ( $n=$ $32)$ or chronic or chronic granulating $(n=39)$. Twenty-three inflammatory cases showed specific histomorphological findings. Schistosomiasis $(n=10)$ was an additional finding in four malignant cases. There were two cases of a cysticercosis. Eleven cases were consistent with tuberculosis. However, only one of them could be confirmed by demonstration of acid-fast bacilli in a $\mathrm{ZN}$ staining, resulting in the need for correlation with microbiological cultures.

Benign tumors and tumor-like lesions

Among the benign tumors, there were 15 epithelial neoplasms, e.g., benign salivary gland tumors like pleomorphic adenomas and ovarian tumors like granulosa cell tumors. Within the group of benign mesenchymal tumors or tumorlike lesions, there were leiomyomas of the uterus, hemangiomas, and spindle cell proliferations of unknown lineage, which could not be finally classified due to lack of immunohistochemistry.

\section{Premalignant lesions}

The vast majority of premalignant lesions consisted of cervical intraepithelial neoplasia (CIN I-III), diagnosed in cervical biopsies, excisions, and three hysterectomy specimens. Two cases showed squamous cell dysplasia of the conjunctiva.

\section{Malignant tumors}

Among the malignant cases were 11 pediatric tumors (patients under 18 years): three cases of small blue round cell tumors (not otherwise classifiable), two retinoblastomas, two cases consistent with rhabdomyosarcoma, and two Kaposi's sarcomas. The large group of adult malignant tumors consisted of 76 cases $(58.9 \%)$ from female patients and 53 cases $(41.1 \%)$ from male patients. The most frequent malignancies among women were cervical squamous cell carcinomas (SCCs; $n=25 ; 32.9 \%$ of all female malignancies), followed by breast carcinomas $(n=12 ; 15.8 \%)$, esophageal 
Fig. 2 Examples of cases. a-c Very large dermatofibrosarcoma protuberance of the abdominal wall (15-year-old male). a Macroscopic top view. $\mathbf{b}$ Macroscopic cut surface. c Histology $(\mathrm{H} \& \mathrm{E} ; \times 2.5)$. d-f Giant dermal nevus with underlying large diffuse neurofibroma (1-year-old girl). d Overview $(\mathrm{H} \& \mathrm{E} ; \times 2.5)$-note chondroid metaplasia (right angle). e Dermal nevus (H\&E, $\times 10)$. f Wagner-Meissner bodies within diffuse neurofibroma (H\&E, $\times 10)$. g Collision of a mature teratoma and a dysgerminoma in the ovary (18year-old female; $\mathrm{H} \& \mathrm{E}, \times 2.5$; inset, magnification of dysgerminoma). h Kaposi's sarcoma of the tonsil region (39year-old female; H\&E, $\times 10$ ). i Colon specimen with granulomatous inflammation in the submucosa due to schistosomiasis (13-year-old girl, with the clinical diagnosis of Hirschsprung's disease;

H\&E, $\times 20$ ). $\mathbf{j}$ Squamous cell carcinoma of the urinary bladder (39-year-old male; H\&E, $\times 20$ ) with schistosoma eggs (inset, magnification)
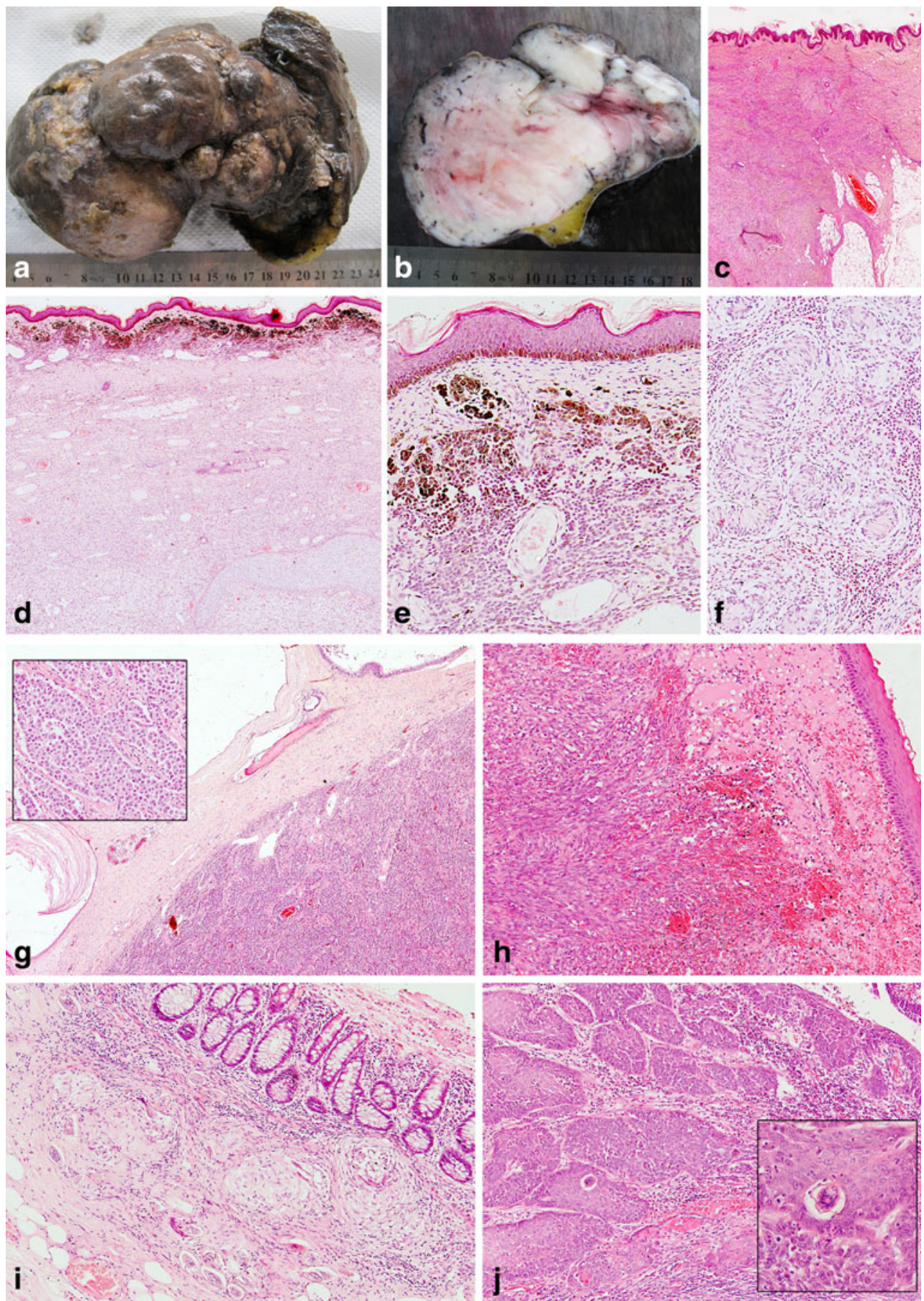

$\operatorname{SCC}(n=9 ; 11.8 \%)$, and ovarian carcinomas $(n=7 ; 9.2 \%)$. Within the group of males, the most frequent malignant tumors were esophageal SCC $(n=9 ; 17.0 \%)$ and SCC of the urinary bladder $(n=7 ; 13.2 \%)$. The median age of adult cancer patients was 43.5 years for females (range, 1887 years) and 52 years for males (range, 24-82 years). Of note, the overall percentage of Kaposi's sarcomas and lymphomas was relatively low, with only six cases $(4.3 \%)$ and seven cases $(5 \%)$, respectively. Other tumors, like laryngeal carcinoma, skin cancer, or intestinal cancers were comparably rare. Metastatic disease was found in ten cases (7.1\%), comprising two metastases of primary gastric cancer and eight metastases without known primary. An overview of the most frequently seen adult malignancies is given in Tables 1 and 2 .

Among the rare or unexpected tumors were (a) a highgrade sarcoma with rhabdoid features from a 23 -year-old female, presenting as a large abdominal mass, (b) a soft tissue manifestation (clinical information) of a chondrosarcoma with extension into the musculus iliopsoas from a 22year-old woman, (c) a dysgerminoma of the ovary of an 18- 
Table 1 Most frequently seen adult female malignancies

\begin{tabular}{llll}
\hline Site & No. & Percentage & $\begin{array}{l}\text { Median age } \\
\text { (min-max) }\end{array}$ \\
\hline Cervix uteri & 23 & 31.9 & $42(28-70)$ \\
Breast & 11 & 15.3 & $50(29-60)$ \\
Esophagus & 9 & 12.5 & $40(32-57)$ \\
Skin & 5 & 6.9 & $46(32-70)$ \\
Ovaries & 5 & 6.9 & $28(18-52)$ \\
Lymphomas & 3 & 4.2 & $30(27-50)$ \\
Total adult female malignancies & 72 & 100 & $43(18-87)$
\end{tabular}

year-old female with a simultaneous mature teratoma, (d) a gigantic dermatofibrosarcoma protuberance from a 15 -yearold boy (without fibrosarcomatous areas), and (e) three cases of malignant melanoma. A number of tumors could not be finally classified due to the inability to determine the tumor's lineage due to the lack of additional immunohistochemical stains or molecular diagnostics (e.g., for the determination of specific translocations).

\section{Discussion}

In low-resource medicine, it is not uncommon for pathologists to be only rarely involved in the diagnostic process during the treatment of patients - in benign or malignant conditions alike. Patients are treated either without a pathologic or laboratory diagnosis based on clinical suspicion alone or are not treated at all. Pathology service in developing countries is complicated by insufficient infrastructural conditions and the lack of specialized personnel and technical equipment [1-3]. Thus, tissue may be submitted for histopathological examinations just in case of clinically highly relevant questions. And even in these cases, clinicians may be faced with long turnover times - often exceeding many months - and the provision of differential diagnoses

Table 2 Most frequently seen adult male malignancies

\begin{tabular}{llll}
\hline Site & No. & Percentage & $\begin{array}{l}\text { Median age } \\
\text { (min-max) }\end{array}$ \\
\hline Esophagus & 9 & 18 & $55(27-79)$ \\
Bladder & 7 & 14 & $39(28-82)$ \\
Soft tissue & 5 & 10 & $54(30-76)$ \\
Lymphomas & 4 & 8 & $36(24-60)$ \\
Larynx & 4 & 8 & $50(26-76)$ \\
Skin & 3 & 6 & $40(25-61)$ \\
Prostate & 3 & 6 & $72(60-76)$ \\
Total adult male malignancies & 50 & 100 & $52(24-82)$ \\
\hline
\end{tabular}

only, especially in those instances when special additional methods would be mandatory to solve the case.

During the 6 weeks, we had the opportunity to gain insight into the diagnostic spectrum of a typical histopathological laboratory in sub-Saharan Africa. A comparison with statistics of a previous year showed that the variety of our cases is representative of the average distribution and character of the annual workload. The majority of biopsies and excisions received consisted of quantitatively adequate, well-preserved tissue, enabling high-quality histomorphology. Fixation of larger resection specimens was sometimes unsatisfactory; however, this did not impede diagnosis. In contrast, fine needle aspiration (FNA), which represents a much simpler technique requiring far fewer resources, failed to provide satisfactory diagnostic material in many cases of our series. This coincides with similar observations from other low-resource countries and highlights the necessity for FNA training facilities.

Probably due to the low rate of submitted specimens mentioned above, the number of cases with significant pathologic findings was remarkably high as compared to a western world pathology laboratory - in particular the percentage of malignancies (34.5 vs. $19.2 \%$ in the Institute of Pathology, Technische Universität München, Germany, during the same time period). It is important to keep in mind that therefore our case series cannot be used to estimate prevalence. Milner et al. are currently preparing a 10-year retrospective paper which covers a full pathoepidemiological assessment of Malawi's pathology from 1997 to 2007.

Communicable diseases, e.g., malaria, HIV, and tuberculosis (including HIV-associated), represent a major cause of morbidity and mortality in central and southeast Africa $[5,6$, 8-10]. In accordance, a significant number of cases were histomorphologically most consistent with tuberculosis or atypical mycobacterial infection. This was seen in about half of the lymph nodes sent in for examination, but also at various other sites like soft tissue or joints. Using ZN staining, the presence of acid-fast bacilli could be confirmed in only one of those cases. However, the $\mathrm{ZN}$ results are most probably false negative due to suboptimal staining procedures, as reported by others in similar settings [11].

Another very interesting finding was the frequency of schistosomiasis, which is widespread in Malawi like in other African countries $[12,13]$. In our cases, schistosoma eggs causing inflammation were found at various sites, e.g., the cervix uteri, the urinary bladder (some as additional finding to carcinoma), or in the appendix, causing an acute perforating appendicitis. Of note, schistosomiasis was the definite histopathological diagnosis in a colon segment of a 13-yearold girl, which was removed with the clinical diagnosis of Hirschsprung's disease, and in a hysterectomy specimen resected due to the clinical impression of a cervical carcinoma. The majority of inflammatory lesions, although 
representing $25 \%$ of the caseload, however, failed to present specific etiologic findings.

In the large group of tumoral conditions, the number of malignant and premalignant cases dominated over benign conditions. Most benign tumors were soft tissue tumors, e.g., leiomyomas of the uterus. There were some rare findings, like a lipofibromatosis/fibrous hamartoma of infancy or a large diffuse neurofibroma underlying a giant dermal nevus, as well as two peripheral ossifying fibromas in the oral cavity.

Although in a number of cases a definitive diagnosis could not be performed due to lack of ancillary techniques, we could at least estimate the dignity in all these cases or provide the most probable differential diagnoses according to the histomorphological picture. Apart from the soft tissue tumors, we also saw a considerable number of benign salivary gland tumors, mainly pleomorphic adenomas $(n=6)$. This is consistent with the observation of the high incidence of salivary gland tumors among African people [14].

Most of the pediatric cases were malignant. Among them are two retinoblastomas and two rhabdomyosarcomas - one of them representing a recurrent case with current "small round cell tumor" morphology. Of note, there were three other cases of small round cell tumors; one of them probably a hematolyphoid malignancy. Unfortunately, the lack of ancillary techniques impeded a definitive diagnosis.

Within the adult malignant cases, the high frequency of SCC of the cervix uteri and intraepithelial precursor lesions reflects the incidence of HPV-induced cervical cancer as the leading malignancy of women in Malawi and most other African countries [15-17]. Cervical cancer has been shown to have a strong association with HIV as a co-driving factor [18]. Unfortunately, the HIV status was only very inconstantly provided in the clinical submission forms, rendering a reliable analysis in this regard fairly impossible. Schistosomiasis of the cervix uteri was relatively common $(n=3)$, and one case showed concomitant SCC. An etiologic correlation has not yet been sufficiently investigated.

A striking finding was the high percentage of malignant squamous lesions at various other sites, like the esophagus, the urinary bladder, or the conjunctiva. Esophageal squamous cell carcinomas were observed in both females and males, in a relatively high frequency and with a low median patient age (45 years). This represents a marked difference as compared to western countries [16]. Several previous studies have highlighted the differing carcinogenesis of esophageal cancer in high-incidence areas like Southeast Asia or Southern Africa [16, 19, 20]. A number of nutritional or environmental factors have been identified - varying dependent on the geographic area investigated [21, 22]. HPV and/or HIV also may have major impact on esophageal carcinogenesis in these countries [23]. However, larger studies with comprehensive epidemiologic and molecular genetic investigations are still missing in this field.
Conjunctival squamous cell carcinoma is also known to occur with increased frequency in Africa. This is likely related to sun exposure and possibly aggravated by viral infections as HIV; however, the exact etiology of this tumor remains unknown [24].

SCC of the bladder is known to be strongly associated with infections by Schistosoma haematobium $[12,16]$. In fact, the presence of parasite eggs was directly demonstrated in one case of SCC of the bladder and in one case with epithelial changes indefinite for dysplasia. Among the other cases, identification of schistosoma eggs was impeded by extensive tissue infiltration by the SCC.

The incidence of breast cancer in Africa is reported to be increasing, although it still remains less frequent than in western countries. In our collection of cases, mammary carcinomas accounted for $15 \%$ of all female malignancies. Breast cancer in Africa has been shown to be unusually aggressive and to affect women at a relatively young age [16, 25-28]. In our collection, however, only two carcinomas were grade III tumors using the Elston and Ellis grading system, and all were specified as invasive ductal (NOS) some of them with an intraductal component. Regarding the age distribution, five women were 40 years and younger, which could be expected in accordance with the data mentioned above. Interestingly, we saw one case of a male breast cancer, which was already described to occur relatively frequently in this part of Africa [29]. Finally, consistent with the low incidence of lung, colorectal, gastric, and prostatic cancers in sub-Saharan Africa [16], we only had three intestinal carcinomas (one of them a carcinoma of the small intestine), three gastric carcinomas or metastases of gastric carcinomas, four prostate carcinomas, and no lung malignancy.

An interesting finding was our low percentage of Kaposi's sarcomas, which are described to occur in a frequency of around $20 \%$ in female and $50 \%$ in male cancer patients in east Africa [16, 17]. Similarly, albeit in lower extent, the frequency of lymphomas among our cases did not reach the expected numbers. This probably represents either sampling bias during our short visit, clinical acumen at making the diagnosis without biopsy, and/or the fact that fewer than $20 \%$ of malignancy diagnoses are being confirmed by laboratory methods (histology, cytology, or hematology) [30].

Unequivocal clinical findings of malignant tumors, like Kaposi's sarcoma in HIV patients or Burkitt's lymphoma in the clinical setting of a rapidly growing mass in association with a dramatic clinical worsening, do not necessarily warrant histopathologic confirmation in the setting of lowresource medicine. The usual therapy of Kaposi's sarcoma consists of antiviral treatment plus a trial and error application of chemotherapy, if available, and diagnosis is confirmed by response [31]. For Burkitt's lymphoma, a rapid 
histopathologic diagnosis would be required, which could be provided only in a very small number of cases [32, 33]. Therefore, treatment is initiated on clinical diagnosis alone.

One has to consider that this is also true in many other cases: even a diagnosis of malignancy would not change therapy due to the lack of adequate therapeutic options. However, in many low-resource countries, medical service improves slowly but steadily as countries progress to higher economic status [34]. Surgical and nonsurgical treatment of oncologic diseases is performed (we saw a case of a breast cancer with the previous history of neoadjuvant chemotherapy). Therefore, adequate pathology services can lead to the recognition of pathology as an integral part of a multidisciplinary healthcare setting. An example is the consensus guideline on breast health care in low-resource and middle-resource countries of the Breast Health Global Initiative, which requires a pathologic diagnosis before the initiation of treatment [35]. Providing pathology service in low-resource countries therefore not only challenges pathologists with difficult working conditions and extraordinary and rare cases, but also provides patient access to better, tailored, and potentially even curative therapies.

There are several modes of support for pathology services: One may be support of already existing laboratories, like the pathologists for Malawi project does, providing immediate load relief for local physicians or covering them during professional education periods. Laboratories, which have been closed due to breakdown of technical equipment and lack of pathologists, can be reestablished [36], and exchange programs can help in forming local pathologists [1]. Teaching clinicians about what can be expected from a pathologic diagnosis can be very helpful, as well as training in performing biopsies and FNAs - the most useful technique in low-resource settings. The immediate feedback given by the pathologists about the quality of the tissue or smears is most often a first, but very important, step for improving quality [37].

Apart from activities by individuals, there are a few organizations, which are coordinating support for pathology in low-resource countries: "Pathologists overseas" (contact: hoenecke57@gmail.com) has been active for almost 20 years [38]. "Pathologists and technicians in favor of developing countries" is a working group within the European Society of Pathology, which mainly consists of members of the Italian organization "Patologi Oltre Frontiera" (www.patologioltrefrontiera.it). They have already performed and are currently conducting several projects in African and Asian countries, e.g., the implementation of telepathology in combination with training of local physicians [11].

In summary, our volunteering work gave us a broad insight into the spectrum of diseases that pathologists can expect in sub-Saharan countries $[39,40]$ and the challenges that pathology is faced with in low-resource settings. While working as volunteering pathologists, both a rich cultural and an intellectually stimulating professional experience can be achieved, at the same time providing a much needed and appreciated diagnostic service.

Acknowledgments The authors thank Kingsley Makwakwa and Fred Chimzimu for excellent technical assistance, and Rob Krysiak and our housekeeper Naomi for taking care of us during our stay in Lilongwe and Blantyre, respectively. We also like to thank Heinz Hoenecke (Pathologists Overseas) for bringing us in contact with the Pathologists for Malawi Project. Furthermore, we thank Katja Specht, MD for expert reevaluation of some tricky cases back at home.

Conflict of interest The authors declare no conflict of interest.

\section{References}

1. Adeyi OA (2011) Pathology services in developing countries - the West African experience. Arch Pathol Lab Med 135(2):183-186. doi:10.1043/2008-0432-CCR.1

2. Benediktsson H, Whitelaw J, Roy I (2007) Pathology services in developing countries: a challenge. Arch Pathol Lab Med 131 (11):1636-1639. doi:10.1043/1543-2165(2007)131[1636:psidca] 2.0.co;2

3. Rambau PF (2011) Pathology practice in a resource-poor setting: Mwanza, Tanzania. Arch Pathol Lab Med 135(2):191-193. doi:10.1043/1543-2165-135.2.191

4. Wikipedia (2012) Malawi. http://en.wikipedia.org/wiki/Malawi. Accessed 10 Jan 2012

5. Mathers CD, Loncar D (2006) Projections of global mortality and burden of disease from 2002 to 2030. PLoS Med 3(11):e442. doi:10.1371/journal.pmed.0030442

6. Mhalu FS (2005) Burden of diseases in poor resource countries: meeting the challenges of combating HIV/AIDS, tuberculosis and malaria. Tanzan Health Res Bull 7(3):179-184

7. Carlson JW, Lyon E, Walton D, Foo WC, Sievers AC, Shulman LN, Farmer P, Nose V, Milner DA Jr (2010) Partners in pathology: a collaborative model to bring pathology to resource poor settings. Am J Surg Pathol 34(1):118-123. doi:10.1097/PAS.0b013e3181c17fe6

8. Jones LA, Chilton JA, Hajek RA, Iammarino NK, Laufman L (2006) Between and within: international perspectives on cancer and health disparities. J Clin Oncol 24(14):2204-2208. doi:10.1200/jco.2005.05.1813

9. Zachariah R, Ford N, Philips M, Lynch S, Massaquoi M, Janssens V, Harries AD (2009) Task shifting in HIV/AIDS: opportunities, challenges and proposed actions for sub-Saharan Africa. Trans R Soc Trop Med Hyg 103(6):549-558

10. WHO (2011) Global tuberculosis control 2011. http://www.who. int/gho/mdg/diseases/hiv/en/index.html. World Health Organization, Geneva, Switzerland http://www.who.int/tb/publications/ global_report/2011/gtbr11_full.pdf

11. Pagni F, Bono F, Di Bella C, Faravelli A, Cappellini A (2011) Virtual surgical pathology in underdeveloped countries: the Zambia project. Arch Pathol Lab Med 135(2):215-219. doi:10.1043/ 1543-2165-135.2.215

12. Ross AGP, Bartley PB, Sleigh AC, Olds GR, Li Y, Williams GM, McManus DP (2002) Schistosomiasis. N Engl J Med 346 (16):1212-1220. doi:10.1056/NEJMra012396

13. Steinmann P, Keiser J, Bos R, Tanner M, Utzinger J (2006) Schistosomiasis and water resources development: systematic 
review, meta-analysis, and estimates of people at risk. Lancet Infect Dis 6(7):411-425. doi:10.1016/S1473-3099(06)70521-7

14. Masanja MI, Kalyanyama BM, Simon EN (2003) Salivary gland tumours in Tanzania. East Afr Med J 80(8):429-434

15. Anorlu RI (2008) Cervical cancer: the sub-Saharan African perspective. Reprod Health Matters 16(32):41-49

16. Jemal A, Bray F, Center MM, Ferlay J, Ward E, Forman D (2011) Global cancer statistics. CA Cancer J Clin 61(2):69-90. doi:10.3322/caac. 20107

17. Parkin DM, Sitas F, Chirenje M, Stein L, Abratt R, Wabinga H (2008) Part I: cancer in indigenous africans - burden, distribution, and trends. Lancet Oncol 9(7):683-692. doi:10.1016/s1470-2045(08)70175-x

18. Chirenje ZM (2005) HIV and cancer of the cervix. Best Pract Res Clin Obstet Gynaecol 19(2):269-276

19. Hendricks D, Parker MI (2002) Oesophageal cancer in Africa. IUBMB Life 53(4-5):263-268. doi:10.1080/15216540212643

20. Walker ARP, Adam F, Walker J, Walker BF (2002) Cancer of the oesophagus in Africans in sub-Saharan Africa: any hopes for its control? Eur J Cancer Prev 11(5):413-418

21. Sammon AM (2007) Carcinogens and endemic squamous cancer of the oesophagus in Transkei, South Africa. Environmental initiation is the dominant factor; tobacco or other carcinogens of low potency or concentration are sufficient for carcinogenesis in the predisposed mucosa. Med Hypotheses 69(1):125-131

22. Sammon AM, Iputo JE (2006) Maize meal predisposes to endemic squamous cancer of the oesophagus in Africa: breakdown of esterified linoleic acid to the free form in stored meal leads to increased intragastric PGE2 production and a low-acid reflux. Med Hypotheses 67(6):1431-1436

23. Patel K, Mining S, Wakhisi J, Gheit T, Tommasino M, Martel-Planche G, Hainaut P, Abedi-Ardekani B (2011) TP53 mutations, human papilloma virus DNA and inflammation markers in esophageal squamous cell carcinoma from the Rift Valley, a high-incidence area in Kenya. BMC Res Notes 4:469. doi:10.1186/1756-0500-4-469

24. Orem J, Otieno MW, Remick SC (2004) AIDS-associated cancer in developing nations. Curr Opin Oncol 16(5):468-476

25. Fregene A, Newman LA (2005) Breast cancer in sub-Saharan Africa: how does it relate to breast cancer in African-American women? Cancer 103(8):1540-1550. doi:10.1002/cncr.20978

26. Burson AM, Soliman AS, Ngoma TA, Mwaiselage J, Ogweyo P, Eissa MS, Dey S, Merajver SD (2010) Clinical and epidemiologic profile of breast cancer in Tanzania. Breast Dis 31(1):33-41

27. Rambau PF, Chalya PL, Manyama MM, Jackson KJ (2011) Pathological features of breast cancer seen in Northwestern Tanzania: a nine years retrospective study. BMC Res Notes 4:214. doi:10.1186/1756-0500-4-214

28. Roy I, Othieno E (2011) Breast carcinoma in Uganda: microscopic study and receptor profile of 45 cases. Arch Pathol Lab Med 135 (2):194-199. doi:10.1043/2008-0421-sor1.1

29. Ravandi-Kashani F, Hayes TG (1998) Male breast cancer: a review of the literature. Eur J Cancer 34(9):1341-1347 (Oxford, England: 1990)
30. Msyamboza KP, Dzamalala C, Mdokwe C, Kamiza S, Lemerani M, Dzowela T, Kathyola D, Parkin DM (2011) Burden of cancer in Malawi; common types, incidence and trends 2007-2010: national population based cancer registry. Paper presented at the 15 th College of Medicine Research Dissemination Conference, Blantyre, Malawi, 15th November 2011

31. Krown SE (2011) Treatment strategies for Kaposi sarcoma in subSaharan Africa: challenges and opportunities. Curr Opin Oncol 23 (5):463-468, 410.1097/CCO.1090b1013e328349428d

32. Ogwang MD, Zhao W, Ayers LW, Mbulaiteye SM (2011) Accuracy of Burkitt lymphoma diagnosis in constrained pathology settings: importance to epidemiology. Arch Pathol Lab Med 135 (4):445-450. doi:10.1043/2009-0443-ep.1

33. Naresh KN, Ibrahim HAH, Lazzi S, Rince P, Onorati M, Ambrosio MR, Bilhou-Nabera C, Amen F, Reid A, Mawanda M, Calbi V, Ogwang M, Rogena E, Byakika B, Sayed S, Moshi E, Mwakigonja A, Raphael M, Magrath I, Leoncini L (2011) Diagnosis of Burkitt lymphoma using an algorithmic approach - applicable in both resource-poor and resource-rich countries. Br J Haematol 154 (6):770-776. doi:10.1111/j.1365-2141.2011.08771.x

34. Farmer P, Frenk J, Knaul FM, Shulman LN, Alleyne G, Armstrong L, Atun R, Blayney D, Chen L, Feachem R, Gospodarowicz M, Gralow J, Gupta S, Langer A, Lob-Levyt J, Neal C, Mbewu A, Mired D, Piot P, Reddy KS, Sachs JD, Sarhan M, Seffrin JR (2010) Expansion of cancer care and control in countries of low and middle income: a call to action. Lancet 376(9747):1186-1193. doi:10.1016/s0140-6736(10)61152-x

35. Anderson BO, Cazap E, El Saghir NS, Yip CH, Khaled HM, Otero IV, Adebamowo CA, Badwe RA, Harford JB (2011) Optimisation of breast cancer management in low-resource and middle-resource countries: executive summary of the Breast Health Global Initiative consensus, 2010. Lancet Oncol 12(4):387-398. doi:10.1016/ S1470-2045(11)70031-6

36. Stalsberg H, Awuah B, Ibarra JA, Nsiah-Asare A (2008) Reestablishing a surgical pathology service in Kumasi, Ghana: case report and discussion of barriers and key elements of a successful collaboration between low- and high-resource countries. Cancer 113(8 Suppl):2338-2346. doi:10.1002/cncr.23830

37. Guggisberg K, Okorie C, Khalil M (2011) Cytopathology including fine-needle aspiration in sub-Saharan Africa: a Cameroon experience. Arch Pathol Lab Med 135(2):200-206. doi:10.1043/ 1543-2165-135.2.200

38. Hoenecke H, Lee V, Roy I (2011) Pathologists overseas: coordinating volunteer pathology services for 19 years. Arch Pathol Lab Med 135(2):173-178. doi:10.1043/2008-0450-sor1.1

39. Bowa K, Wood C, Chao A, Chintu C, Mudenda V, Chikwenya M (2009) A review of the epidemiology of cancers at the University Teaching Hospital, Lusaka, Zambia. Trop Dr 39(1):5-7. doi:10.1258/td.2008.070450

40. Hutt MS, Spencer H (1982) Histopathology services for developing countries. Br Med J (Clin Res Ed) 285(6351):1327-1329 